\title{
Thermoelectric power in one-dimensional Hubbard model
}

\author{
M. M. Zemljič ${ }^{1}$ and P. Prelovšek ${ }^{1,2}$ \\ ${ }^{1}$ J. Stefan Institute, SI-1000 Ljubljana, Slovenia and \\ ${ }^{2}$ Faculty of Mathematics and Physics, University of Ljubljana, SI-1000 Ljubljana, Slovenia
}

(Dated: June 14, 2018)

\begin{abstract}
The thermoelectric power $S$ is studied within the one-dimensional Hubbard model using the linear response theory and the numerical exact-diagonalization method for small systems. While both the diagonal and offdiagonal dynamical correlation functions of particle and energy current are singular within the model even at temperature $T>0, S$ behaves regularly as a function of frequency $\omega$ and $T$. Dependence on the electron density $n$ below the half-filling reveals a change of sign of $S$ at $n_{0}=0.73 \pm 0.07$ due to strong correlations, in the whole $T$ range considered. Approaching half-filling $S$ is hole-like and can become large for $U \gg t$ although decreasing with $T$.
\end{abstract}

PACS numbers: 71.27.+a, 71.10.Fd, 72.15.Jf

\section{INTRODUCTION}

The thermoelectric power (TEP) or the Seebeck coefficient $S$ can show quite anomalous behavior in metals with strongly correlated electrons. This has been recognized very clearly in the normal-state of high- $T_{c}$ cuprates, where numerous experimental studies [1, 2] reveal several unusual features of the TEP $S(T)$ : a) In the underdoped regime with small concentration of holes $n_{h} \ll 1$ introduced into the reference antiferromagnet (AFM) $S$ is positive and large, showing a decrease with temperature $d S / d T<0$, except at very low $T \sim T_{c}$ where the usual Fermi-liquid (FL) behavior $S \propto T$ sets in, b) as a function of hole doping the TEP $S$ also decreases and approaches zero or even changes sign in the overdoped regime. The latter phenomena can be attributed to the effect of strong correlations, since for noninteracting (or weakly interacting) electrons one would expect negative and small $S<0$ within the same regime.

Although one of the easiest transport quantities to measure, the TEP proved to be a hard challenge for a theoretical consideration and understanding. Apparently, it is even more demanding than some other transport properties as, e.g., the electrical conductivity $\sigma$, which are also anomalous in many correlated systems, and in cuprates in particular. Since the Boltzmann transport theory can fail completely for strongly correlated electrons, the general linear response approach has to be followed for $S$ [3]. Nontrivial expressions have been obtained in this way in the limit of high temperature [4] where $S(T \rightarrow \infty)$ approaches a finite value. The limiting value of $S$ is also known for the extended Hubbard model with an infinite-repulsion $U \rightarrow \infty$ [4] and for more more complicated models [5]. An extension of $S(T)$ to finite $T<\infty$ has been studied within the $U=\infty$ Hubbard model using the retraceable-path approximation [6]. A numerical analysis of $S(T)$ within the planar $t-J$ model has obtained several features relevant to cuprates [7]. The latter calculation is based on an observation that the ratio of off-diagonal and diagonal dynamical correlation functions between the energy and particle current, respectively, is nearly constant. Using the exact diagonalization of small systems the TEP has been studied also for models with the orbital degeneracy [8]. Another line of approach is to express $S(T)$ in terms of spectral functions. This is well posed approximation within the dynamical meanfield theory [9, 10], valid for the Hubbard model in the limit of large dimensions. Using spectral functions obtained via the fluctuation-exchange approximation the latter approach has been applied also to the $2 \mathrm{D}$ model [11] and further improved by the inclusion of vertex corrections [12].

The one-dimensional (1D) Hubbard model, which is the subject of this study, is known to possess special features relative to systems with higher $D>1$. As 1D models of interacting electrons in general, it complies to the phenomenology of Luttinger liquids and exhibits the spin-charge separation [13]. Using the Bethe-Ansatz solution for low-energy spinon and holon excitation branches, the low- $T$ behavior of $S$ has been considered in Ref.[14]. Results appear quite unusual since $S$ is claimed to remain positive at all electron densities $n<1$ below half filling. This study indicates that even $S(T \rightarrow 0)$ is an open question within the 1D Hubbard model, and even more the behavior at higher $T$. On the other hand, it has been recognized that the integrability of the 1D Hubbard model implies singular transport quantities [15, 16, 17]. In particular, it has been shown that away from half-filling, i.e., for $n \neq 1$, the charge and energy (heat) transport remain dissipationless even at $T>0$, i.e., in the d.c. limit the electrical as well as the thermal conductivity both diverge, $\sigma_{0} \rightarrow \infty$ and $\lambda_{0} \rightarrow \infty$ [17]. Since $S(T)$ is the ratio of two correlation functions, it is expected to remain well defined and finite [15].

In order to clarify the behavior of the TEP within the 1D Hubbard model, we present in the following results of the numerical study of the dynamical $\tilde{S}(\omega, T)$, based on the linear response theory, generalized to allow for singular transport quantities. Dynamical response functions are evaluated numerically for finite chains with $L$ sites using the approach of exact diagonalization. In particular, for the largest systems we apply the finite-temperature Lanczos method (FTLM) [7] which enables us to consider a model with up to $L=14$ sites . We concentrate on the regime of strong correlations $U \geq 2 t$.

The paper is organized as follows: In Sec. 2 we present the general linear response formalism for transport quantities 
within the 1D Hubbard model, with the special care for singular behavior in the d.c. limit $\omega \rightarrow 0$. Different limits are also considered. Results of the numerical study of finite systems are presented in Sec. 3 and conclusions are given in Sec. 4.

\section{LINEAR RESPONSE THEORY}

We consider the 1D Hubbard model

$$
H=-t \sum_{l, s}\left(c_{l+1, s}^{\dagger} c_{l, s}+\text { H.c. }\right)+U \sum_{l} n_{l \uparrow} n_{l \downarrow},
$$

with the nearest neighbour (n.n.) hopping term $H_{t}$ and the onsite repulsion $U$ on a chain of $L$ sites. The particle current has the usual form (we set further $\hbar=1$ and the lattice spacing $a_{0}=1$ ),

$$
J_{n}=t \sum_{l, s}\left(i c_{l+1, s}^{\dagger} c_{l s}+\text { H.c. }\right)
$$

whereas the energy current can be expressed in terms of n.n. energy operators [17, 18]

$$
J_{E}=i \sum_{l}\left[h_{l}, h_{l+1}\right], \quad H=\sum_{l} h_{l} .
$$

It then follows [17] from Eq. (1)

$$
\begin{aligned}
& J_{E}=-t^{2} \sum_{l, s}\left(i c_{l+1, s}^{\dagger} c_{l-1, s}+\text { H.c. }\right)+ \\
& +\frac{U t}{2} \sum_{l, s}\left\{i\left(c_{l+1, s}^{\dagger} c_{l s}+c_{l s}^{\dagger} c_{l-1, s}\right) n_{l,-s}+\text { H.c. }\right\} .
\end{aligned}
$$

Within the linear response formalism we calculate general transport coefficients $L_{i j}$ [3], defined by $J_{i}=\sum_{j} L_{i j} F_{j}$. Here, $i, j=n, Q$, where $J_{Q}=J_{E}-\mu J_{n}$ is the heat current, $\mu$ is the chemical potential, and related generalized forces are given by $F_{n}=-\left(\nabla \mu+e_{0} \mathcal{E}\right) / T, F_{Q}=-\nabla T / T^{2}$. Since the transport coefficients in the 1D Hubbard model are singular in the d.c. limit [15, 17], it is essential to generalize the formalism to finite frequencies, dealing with response functions $L_{i i}(\omega)$. If one introduces appropriate polarization operators [19. 20] $P_{n}=\sum_{l} l n_{l}, P_{E}=\sum_{l} l h_{l}$ and $P_{Q}=P_{E}-\mu P_{n}$ (valid for open boundary conditions), we can write within the linear response theory

$$
L_{i j}(\omega)=\frac{T}{i \omega^{+}}\left(\left\langle\tau_{i j}\right\rangle-\chi_{i j}(\omega)\right)
$$

where $\omega^{+}=\omega+i \epsilon$,

$$
\tau_{i j}=-\frac{i}{L}\left[J_{i}, P_{j}\right]
$$

and

$$
\chi_{i j}(\omega)=-\frac{i}{L} \int_{0}^{\infty} d t e^{i \omega^{+} t}\left\langle\left[J_{i}(t), J_{j}(0)\right]\right\rangle
$$

It follows from Eq. (6] that $\tau_{n n}=-H_{t} / L$ [20], and

$$
\begin{aligned}
& \tau_{n E}=\frac{2 t^{2}}{L} \sum_{l, s}\left(c_{l+1, s}^{\dagger} c_{l-1, s}+\text { H.c. }\right)- \\
& -\frac{U t}{2 L} \sum_{l, s}\left\{\left(c_{l+1, s}^{\dagger} c_{l s}+c_{l s}^{\dagger} c_{l-1, s}\right) n_{l,-s}+\text { H.c. }\right\},
\end{aligned}
$$

while $\tau_{n Q}=\tau_{n E}-\mu \tau_{n n}$. Note that the dynamical electrical conductivity, more frequently discussed in this context, is given by $\sigma(\omega)=e_{0}^{2} L_{n n}(\omega) / T$.

Finally, we get for the dynamical TEP [3]

$$
\begin{aligned}
\tilde{S}(\omega) & =-\frac{1}{e_{0} T} \frac{L_{n Q}(\omega)}{L_{n n}(\omega)}= \\
& =-\frac{1}{e_{0} T}\left(\frac{\left\langle\tau_{n E}\right\rangle-\chi_{n E}(\omega)}{\left\langle\tau_{n n}\right\rangle-\chi_{n n}(\omega)}-\mu\right) .
\end{aligned}
$$

In most model systems (and in real materials) we are dealing with the situation where transport coefficients at $T>0$ are not singular within the d.c. limit, i.e., $L_{i j}(\omega \rightarrow 0)=L_{i j}^{0}$ are finite. This is related to the ergodic behavior of the system and Eq. (5) in this case requires the equality of the d.c. adiabatic and isothermal susceptibilities, $\chi_{i j}(\omega \rightarrow 0)=\chi_{i j}^{T} \equiv\left\langle\tau_{i j}\right\rangle$ [19].

It has been shown that in this respect the 1D Hubbard model is singular [17], as well as some other 1D integrable quantum many-body models [15]. Let us define the generalized stiffness

$$
D_{i j}=\frac{1}{2}\left(\left\langle\tau_{i j}\right\rangle-\chi_{i j}(\omega \rightarrow 0)\right)
$$

Within the 1D Hubbard model $D_{i j}(T>0)$ remain finite away from half-filling $n \neq 1$ [17]. The general argument has been given with the finite overlap of current operators $\left\langle J_{i} Q_{n}\right\rangle$ where $Q_{n}, n=1, L$ are conserved quantities due to integrability. Note that in a macroscopic system there is an infinite number of operators $Q_{n}$. In particular, $Q_{3}$ corresponds closely to $J_{E}$, obtained by the replacement in Eq.(4) $U / 2 \rightarrow U$. In spite of singular $L_{i j}(\omega \rightarrow 0)$ the d.c. TEP $S=\tilde{S}(\omega \rightarrow 0)$ is expected to remain well defined and finite. From the representation chosen in Eq. (10) we can express $S$ as

$$
S=-\frac{1}{e_{0} T}\left(\frac{D_{n E}}{D_{n n}}-\mu\right) .
$$

There are some limits which give a nontrivial test to our results presented below. For noninteracting electrons at $T>0$ it is straightforward to evaluate $S$. Since $J_{n}$ and $J_{E}$ are conserved quantities (constants of motion), we get from Eq. (7) that $\chi_{n n}(\omega)=\chi_{n E}(\omega)=0$ and

$$
\begin{aligned}
\tau_{n n} & =\int f(\epsilon) g(\epsilon) \epsilon d \epsilon, \\
\tau_{n E} & =\int f(\epsilon) g(\epsilon)\left(2 \epsilon^{2}-4 t^{2}\right) d \epsilon,
\end{aligned}
$$


where $g(\epsilon)=2\left(4 t^{2}-\epsilon^{2}\right)^{-1 / 2} / \pi, f=1 /(\exp [(\epsilon-\mu) / T]+1)$ and $\mu$ is fixed by the density $n=\int f(\epsilon) g(\epsilon) d \epsilon$.

In the limit of large $k_{B} T \gg(U, t)$ the result coincides with that of noninteracting electrons at $k_{B} T \gg t$,

$$
S=-\frac{k_{B}}{e_{0}} \ln \frac{2-n}{n} .
$$

On the other hand, a nontrivial generalized Heikes formula is obtained for the case of large $\left(k_{B} T, U\right) \gg t$ but $k_{B} T \ll U$ [4],

$$
S=-\frac{k_{B}}{e_{0}} \ln \frac{2(1-n)}{n} .
$$

It has been recently observed [7] that in the most frustrated regime of strongly correlated systems the transport coefficients $L_{n E}$ and $L_{n n}$ are closely related. Numerical results for the planar $t-J$ model thus reveal (at the intermediate doping of the reference antiferromagnet) that in a broad range of $\omega, T$ the ratio $R(\omega)=L_{n E}(\omega) / L_{n n}(\omega)$ is nearly constant. Extending this behavior to $T \rightarrow 0$ a simple relation for $S(T)$ would follow, which is very attractive for application in complicated correlated systems [21]. It is of interest to which extent within the 1D Hubbard model $R(T)=D_{n E} / D_{n n}$ behaves similarly.

\section{RESULTS}

We analyse dynamical transport coefficients $L_{n n}(\omega)$ and $L_{n E}(\omega)$ and consequently $\tilde{S}(\omega)$ by performing the exact diagonalization of the model Eq. (1) on a finite chain with $L$ sites and with periodic boundary conditions. Taking into account the number of electrons $N_{e}$ and the total spin projection $S^{z}$ as conserved quantities as well as the translation symmetry (wavevector $q$ ) systems up to $L=10$ sites (all fillings) can be fully diagonalized to get results at arbitrary $T$. Using the FTLM one can analyse larger systems at $T>0$ [7] and in the following we present results obtained for $L=12,14$ with the number of basis states within a symmetry sector up to $N_{s t} \sim 800.000$. All $N_{e} \leq L$ (for $N_{e}>L$ we employ the electron-hole symmetry of the model) are investigated in order to perform the grand-canonical averaging which allows for a continuous variation of $n=N_{e} / L$. In the present FTLM application we use typically $M=100$ Lanczos steps and random averaging over $R \sim 20$ samples within each symmetry sector.

Within FTLM, as well as in any exact calculation of a small system, it is important to realize that results have a restricted thermodynamic validity due to finite-size effects, which begin to dominate at low $T<T_{f s}$. Clearly, $T_{f s}$ depends on the size of the system, but as well on the density and character of low- $T$ excitations. It is characteristic that $k_{B} T_{f s} / t \sim 0.2$ (at $n \sim 1$ ) in the 1D Hubbard chain with $L=14$ is larger that in a corresponding 2D (square lattice) Hubbard model with $N=4 \times 4=16$ sites [22] (where it was found $k_{B} T_{f s} / t \sim 0.1$ at the intermediate doping of the reference insulator) in spite of an evidently shorter linear extension. The difference can be explained with the anomalous behavior of the planar Hubbard model (in the strong-correlation regime $U \gg t$ ) [22] and of related 2D $t$ - $J$ model [7] which show a non-FL behavior close to half-filling, i.e., a large degeneracy of low-lying states results in a large entropy and small $T_{f s}$. On the other hand, the 1D Hubbard model at low $T$ follows the phenomenology of Luttinger liquids [13], with well defined excitation branches of spinons and holons, resulting in an increased but not anomalous entropy.

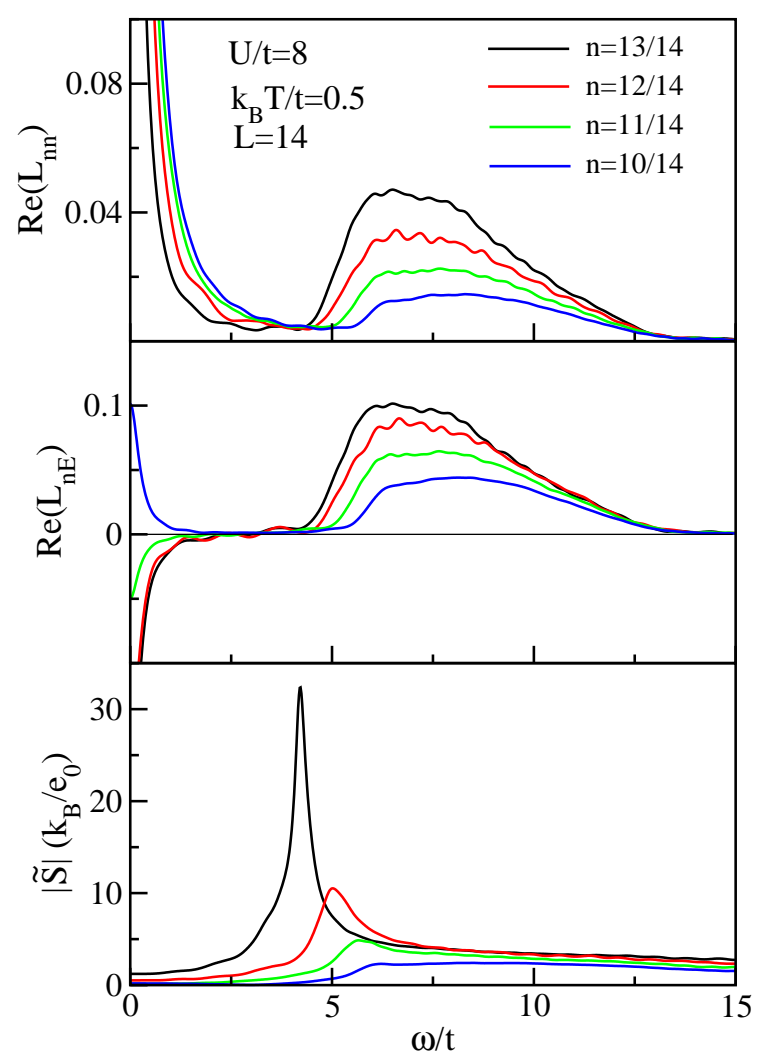

FIG. 1: Transport coefficients $\operatorname{Re} L_{n n}(\omega), \operatorname{Re} L_{n E}(\omega)$ and TEP $|\tilde{S}(\omega)|$, calculated within the 1D Hubbard model with $U / t=8$, $L=14, T=0.5 t$, for different electron densities $n$ close to halffilling. Results obtained via FTLM are broadened with $\delta=0.25 t$.

Let us first discuss the frequency-dependent $L_{n n}, L_{n E}$ and $\tilde{S}$. In Fig. 1 we present $\operatorname{Re} L_{n n}(\omega) \propto \sigma, \operatorname{Re} L_{n E}(\omega)$ as well as $|\tilde{S}(\omega)|$, calculated using the FTLM in a $L=14$ system for the typical strong-correlation regime $U=8 t$ for fixed $k_{B} T=0.5 t$ and different $n$ close to half-filling. Spectra are smoothed with an additional broadening $\delta=0.25 t$. As already found in previous studies [15, 17], finite $D_{n n}(T>0)$ (away from half-filling $n \neq 1$ ) is reflected in a singular (deltafunction) contribution to $\operatorname{Re} L_{n n}(\omega \rightarrow 0)$ in Fig. 1. The incoherent part pronounced for $\omega>4 t$, mainly due to the transition from the lower to upper Hubbard band, decreases 
away from $n \sim 1$, consistent with the free electron propagation when approaching the empty band, $n \rightarrow 0$. An analogous structure is observed in the off-diagonal $L_{n E}(\omega)$, whereby the singular contribution $D_{n E}$ can be negative as well, and in fact shows the change of sign upon decreasing $n$. The resulting (absolute value) $|\tilde{S}(\omega)|$ evaluated from Eq. (9) is presented in Fig. 1. In the strong-correlation regime it seems to be characteristic (close to half-filling) that $\tilde{S}(\omega)$ shows a pronounced resonance at $\omega \sim \omega_{0}$ with $\omega_{0}$ linked to the pseudo-gap in the incoherent part. The resonance is determined by the minimum of $\left|L_{n n}\left(\omega_{0}\right)\right|$, i.e., by the vanishing $\operatorname{Im} L_{n n}\left(\omega_{0}\right)=0$ whereby also $\operatorname{Re} L_{n n}\left(\omega_{0}\right)$ is small. This results in a sharp peak in $|\tilde{S}(\omega)|$, with a simultaneous change of phase in complex $\tilde{S}(\omega)$. The same effect we find for $U=4 t$. Away from $\omega \sim \omega_{0}$ the dynamical TEP $|\tilde{S}(\omega)|$ behaves quite smoothly approaching both (different) nontrivial limits $\omega=0$ and $\omega \rightarrow \infty$.

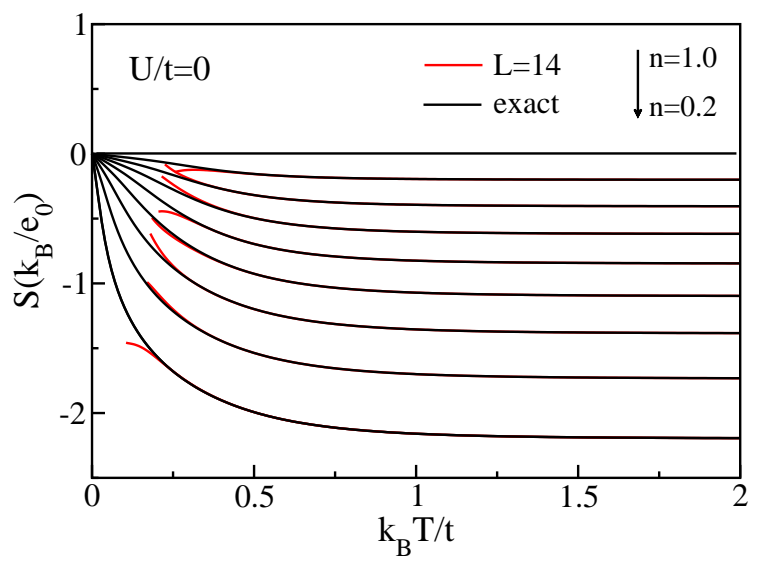

FIG. 2: TEP $S$ (in units $k_{B} / e_{0}$ ) vs. $T$ for noninteracting electrons for different densities $n$, calculated from the analytical result 1211, compared to the result for a small system $L=14$.

In Fig. 2 we present the reference d.c. TEP $S(T)$ for noninteracting fermions, evaluated from Eq. 12111 for different $n$. We also display the result of a grand-canonical calculation of $S$ on a finite-size system with $L=14$ sites. The deviations of the latter from exact results at low $T$ can serve as an estimate for $T_{f s}$, relevant for FTLM calculations presented below. It should be noted that in general at $T \rightarrow 0$ small-system results for $S$ do not get a meaningful limit, in particular they do not reproduce correct FL $S \propto T$ behavior. It follows from Fig. 2 that $k_{B} T_{f s} \sim 0.2-0.3 t$, depending on $n$. Note also that $S \equiv 0$ for $n=1$ due to the particle-hole symmetry of the model, which remains valid also for general $U \neq 0$ [23]. It should also be observed from Fig. 2 that the FL regime with $S \propto T$ is restricted to quite low $k_{B} T<0.4 t$ even for noninteracting fermions, in spite of much broader band $W=4 t$.

FTLM results for $S(T)$ for different $U / t=2,4,8$ and $n=$ $0.3-0.9$ are presented in Fig. 3. For a given system of $L$ sites and fixed $T$ we calculate using FTLM $S(\omega \rightarrow 0)$ values for all sectors $1<N_{e}<L$ (taking into account the symmetry of the

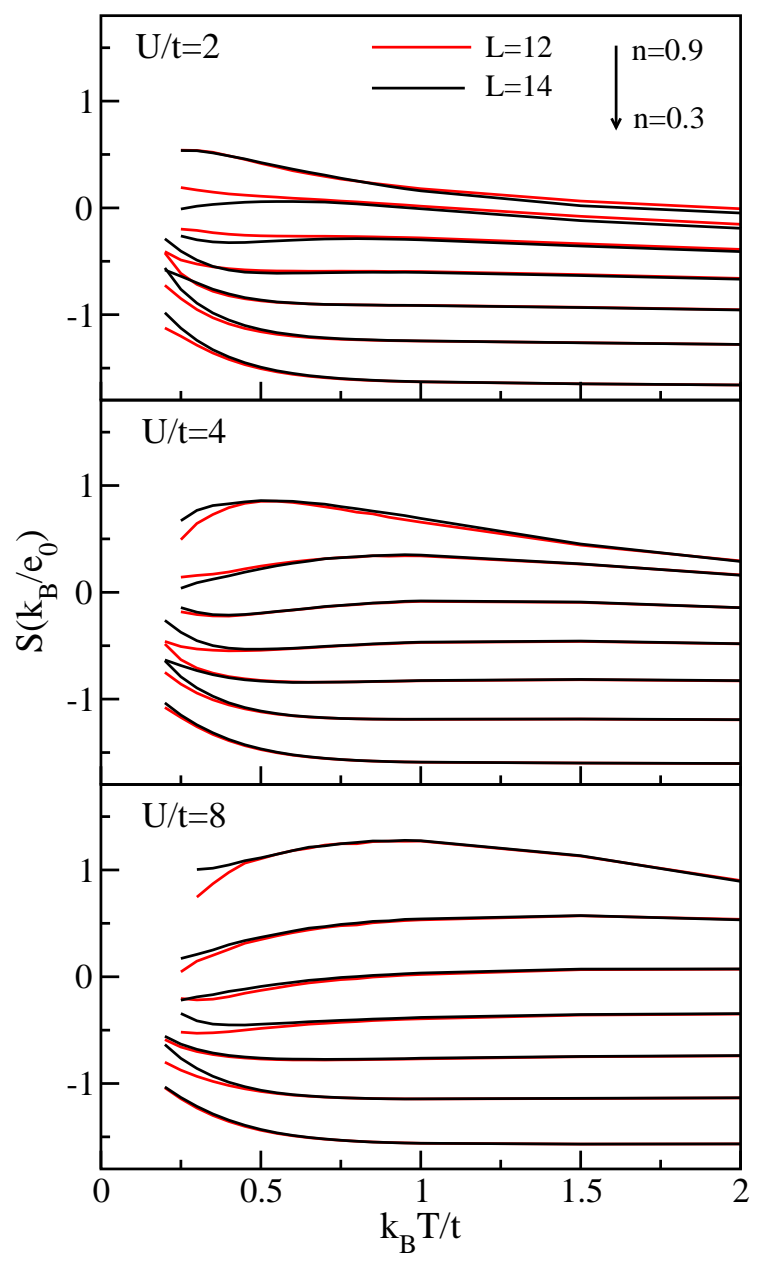

FIG. 3: $S$ vs. $T$ for the 1D Hubbard model with $U / t=2,4,8$, respectively, as calculated for different $n$ with the FTLM for $L=$ 12,14 chains.

model for $L+1<N_{e}<2 L-1$ ) and perform grand-canonical averaging to get a continuous variation $S(T, n)$. We show in Fig. 3 results for two systems, $L=12,14$, which allow to estimate $T_{f s}$ for $U>0$ cases.

Several observations can be made on the basis of $S(T)$ results in Fig. 3: a) For $n \leq 0.6$ we get for all considered $U / t$ $S(T)<0$, with values essentially identical to that of noninteracting electrons in Fig. 2. b) The effect of $U>0$ becomes well pronounced close to half-filling, where the TEP becomes hole-like, i.e. $S>0$, and at the same time large. At large $U \gg t$, the TEP $S(T)$ stays constant in a large $T$ window, whereas for smaller $U$, e.g. $U / t=2$, it is suppressed already for modest $k_{B} T \sim t$ to the noninteracting value $S \sim 0$. c) It is rather difficult to reach the FL regime where $S \propto T$. Still, away from half-filling, i.e. for $n<0.6$, numerical results appear consistent with the behavior $S(T \rightarrow 0) \rightarrow 0$. This is, however, not evident for $n \sim 1$, in particular not for largest $U=8 t$.

In Fig. 4 we display the alternative representation of the TEP results $S(n)$ (for $L=14$ system only) for various 


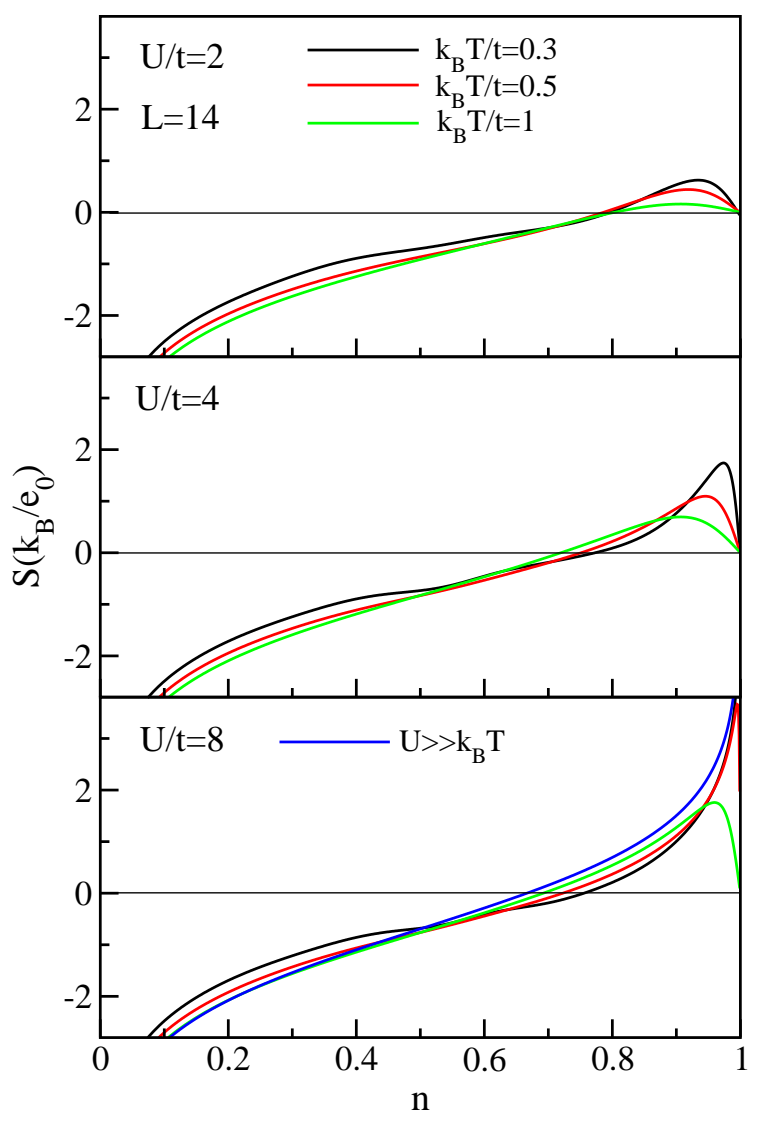

FIG. 4: $S$ vs. $n$ for $U / t=2,4,8$ and different $T$, as calculated with FTLM for $L=14$ chains. For $U / t=8$ case we present also the Heikes value, Eq. 14.

$k_{B} T / t=0.3,0.5,1.0$ and same $U / t$ as in Fig. 3. Again, for $n<0.6$ the TEP is electron-like $S<0$ and behaves as for noninteracting electrons. The interesting part is for $n>0.6$ where $U>0$ induces a transition to a hole-like $S>0$ for $n>n_{0}$. In general, $n_{0}$ is dependent both on $T$ and $U$. The limiting value, reached for $U \gg k_{B} T \gg t$, is given by the generalized Heikes formula, Eq.(14), with the value $n_{0}=2 / 3$. Moreover, as seen in Fig. $4, n_{0}$ remains nearly constant, i.e. $n_{0} \sim 0.7$, in a broad range of $T$ in the strong-correlation regime $U / t \geq 4$. Only for a weak repulsion $U / t=2$ case the crossing starts to approach the noninteracting $n_{0}=1$. Since at $n=1$ we get $S=0$ for all $T$, there must be a maximum $S^{*}=S\left(n=n^{*}\right)$ within the holelike regime. The location $n^{*}$ as well as the value $S^{*}$ depends strongly on $U$ and $T$. The limit, Eq. 114, gives $n^{*}=1$ with divergent $S^{*} \rightarrow \infty$. For $U=8 t$ our results for $k_{B} T \leq 0.5 t$ show a good overall agreement with the latter limiting behavior. Note that here due to large charge gap at $U=8 t$, the maximum appears very close to half-filling, i.e., $n^{*} \rightarrow 1$ with $S^{*} \gg k_{B} / e_{0}$. With decreasing $U / t$ as well as with increasing $T$ the maximum becomes less pronounced and moves away from $n^{*}=1$.

\section{CONCLUSIONS}

Above we have presented results for the dynamical and d.c. TEP within the 1D Hubbard model, as calculated by using the FTLM for small systems with up to $L=14$ sites. Several comments and conclusions are in order:

a) The main restriction in the validity of results comes from the use of small systems. Finite-size effects in $S$ and other quantities start to dominate results for $T<T_{f s}$. Although the FTLM algorithm involves also the random sampling over initial configurations [7], it does not essentially increase the numerical error of $S$ and only slightly increases $T_{f s}$.

b) It is quite characteristic that observed $T_{f s}$ is weakly dependent on $U$ and $n$, i.e., $k_{B} T_{f s} / t \sim 0.2-0.3$ for $L=14$. This is strikingly different from the Hubbard model (or $t-J$ model) in $2 \mathrm{D}$, where $T_{f s}$ varies substantially with concentration $n$ in the strong-correlation regime [7, 22], i.e., $T_{f s}$ has a minimum at the 'optimum' doping where the quantum antiferromagnet is frustrated by mobile holes. The latter regime is characterized by the large entropy, hence also low $T_{f s}$. In the 1D Hubbard model there seems to be no such phenomenon, consistent with the low density of excitations (spinons and holons) within the Luttinger liquid.

c) We have investigated only the regime below half-filling $n<1$, since due to the particle-hole symmetry of the model, Eq. (1), it follows $S(2-n)=-S(n)$.

d) Independent of $U$ at low filling $n<0.6$ the TEP is electronlike, $S<0$, and essentially equal to that of noninteracting electrons in the whole regime of $T$ considered. Although the result appears plausible, it contradicts the conclusion based on the exact Bethe-Ansatz solutions [14] that for large $U>t$ the TEP is hole-like $S>0$ for all $n<1$.

e) TEP becomes strongly dependent on $U$ as well as on $T$ when approaching the half-filling. In particular, it changes the sign at $n_{0}$, which in a broad regime of $U, T$ stays close to the Heikes result $n_{0} \sim 2 / 3$, but increases towards $n_{0} \rightarrow 1$ with the vanishing $U \rightarrow 0 . S(n)$ reaches a maximum at $n=n^{*}$, located in the regime $n_{0}<n^{*}<1$. Both $n^{*}$ and $S^{*}$ are dependent on $U$ and $T$. In particular, $S^{*}$ can become very large in the strong-correlation regime $U \gg t$ where $n^{*} \rightarrow 1$ and $S\left(n<n^{*}\right)$ follows Eq. (14) down to low $T$.

f) The low- $T$ variation of the TEP is expected to follow the standard FL behavior $S \propto T$. It appears quite difficult to enter and investigate the latter regime using small-system diagonalization. The linear dependence is restricted to a rather narrow window $k_{B} T<0.5 t$ even for free fermions, as seen in Fig. 2. For $U \gg t$ close to the half-filling this window is expected to become even narrower, since the relevant energy scale is that of spinons with the characteristic exchange coupling $J=4 t^{2} / U$. So it is not surprising that our results in Fig. 3 for largest $U=8 t$ at $n \sim 1$ do not reveal the onset of the linear regime, down to $k_{B} T_{f s} \sim 0.3 t$. In any case, the low- $T$ behavior of the TEP remains an open question which could be possibly settled by an analytical calculation [14].

g) It is a relevant question to what extent the behavior within 
1D Hubbard model reflects the general features of transport and of TEP in higher dimensional systems with strongly correlated electrons. Of particular interest is the relation with the physics of quasi-2D high- $T_{c}$ cuprates and with their anomalous electronic properties [1, 2], which have been the motivation of the intensive research of microscopic models of correlated electrons. Apart from results based on the approximation of infinite dimensions [9, 10], there are few model calculations of $S$ in 2D systems [7] and the question of the variation of $S(T)$ in prototype models is not settled. Still, some differences and similarities between 1D and higher D systems seem to be evident. Transport in 1D Hubbard model is specific due to its integrability, showing in the divergence of d.c. coefficients $L_{i j}(\omega \rightarrow 0)$. Nevertheless, the TEP $S$ is well behaved and even some deviation from integrability (e.g., by adding additional next n.n. hopping in $H$ ) should not influence its behavior dramatically. The difference between 1D and 2D (or higher $\mathrm{D}$ ) models is in the nature of low-lying excitations. While in the 1D model the latter follow the scenario of the Luttinger liquid, the situation in $2 \mathrm{D}$ is more involved and not fully understood yet. At least numerical investigation reveal a very degenerate quantum state close to optimum doping [7, 22]. One consequence appears to be nearly $\omega$ and $T$-independent ratio $R=L_{n E} / L_{n n}$ [7]. In contrast, in 1D model $R=D_{n E} / D_{n n}$ remains rather $T$-dependent (roughly as much as $\mu$ ), so $S$ cannot be well expressed solely in terms of $\mu(T)$.

h) Nevertheless, there are important similarities between the TEP in 1D Hubbard model and available 2D results [7] and moreover experimental results within the normal state of cuprates [1, 2]. In the strong correlation regime $U \gg t$, $S$ becomes hole-like for $n \leq 1$. In a broad range of $T$ the change of sign appears at $n_{0}=0.73 \pm 0.07$, close to the limiting Heikes value $n=2 / 3$. At the same time the maximum value $S^{*}>0$ can become large and remains as such down to quite low $T$, giving the TEP in strongly correlated system also the potential applicability. At the same time, in the hole-like regime the $T$ dependence of the TEP is quite pronounced. In particular, $d S / d T<0$ at $T>T^{*}$, also consistent with experiments on cuprates, although $T^{*}$ appears higher than in 2D systems [7].

Authors acknowledge the support of the Ministry of Education, Science and Sport of Slovenia under grant Pl-0044.

[1] A. B. Kaiser and C. Uher, in studies of High Temperature Superconductors, Vol.7, Ed. A. V. Narlikar (Nova, New York), p. 353 (1991).

[2] J. R. Cooper and J. W. Loram, J. Phys. I (France), 6, 2237 (1996).

[3] G. D. Mahan, Many-Particle Physics, (Plenum, New York 1990).

[4] P. M. Chaikin and G. Beni, Phys. Rev. B 13, 647 (1976).

[5] W. Koshibae, K. Tsutsui, and S. Maekawa, Phys. Rev. B 62, 6869 (2000).

[6] A. Oguri and S. Maekawa, Phys. Rev. B 41, 6977 (1990).

[7] J. Jaklič and P. Prelovšek, Adv. Phys. 49, 1 (2000).

[8] W. Koshibae and S. Maekawa, Phys. Rev. Lett. 87, 236603 (2001).

[9] G. Palsson and G. Kotliar, Phys. Rev. Lett. 80, 4775 (1995).

[10] V. S. Oudovenko and G. Kotliar, Phys. Rev. B 65, 075102 (2002).

[11] G. Hildebrand, T. J. Hagenaars, W. Hanke, S. Grabowski, and J. Schmalian, Phys. Rev. B 56, R4317 (1997).

[12] H. Kontani, J. Phys. Soc. Jpn. 70, 2840 (2001).

[13] H. J. Schulz, Int. J. Mod. Phys. B 5, 57 (1991).

[14] C. A. Stafford, Phys. Rev. B 48, 8430 (1993).

[15] for a review see X. Zotos and P. Prelovšek in Interacting Electrons in Low Dimensions, Eds. D. Baeriswyl and L. de Giorgi, Kluwer, to appear; also cond-mat/0304630

[16] H. Castella, X. Zotos, and P. Prelovšek, Phys. Rev. Lett. 74, 972 (1995).

[17] X. Zotos, F. Naef and P. Prelovšek, Phys. Rev. B 55, 11029 (1997).

[18] G. Beni, Phys. Rev. B 10, 2186 (1974).

[19] K. Louis and C. Gros, Phys. Rev. B 67, 224410 (2003).

[20] P. F. Maldague, Phys. Rev. B 16, 2437 (1977).

[21] T. Tohyama, S. Maekawa, and P. Prelovšek, Phys. Rev. B 67, 180502(R) (2003).

[22] J. Bonča and P. Prelovšek, Phys. Rev. B 67, 085103(R) (2003).

[23] G. Beni and C. F. Coll, Phys. Rev. B 11, 573 (1975). 